\title{
Recommendations on the Use of Ultrasound Guidance for Adult Thoracentesis: A Position Statement of the Society of Hospital Medicine
}

\author{
Ria Dancel, $\mathrm{MD}^{1 *}$, Daniel Schnobrich, MD², Nitin Puri, MD³, Ricardo Franco-Sadud, MD, Joel Cho, MD, \\ Loretta Grikis, MLS ${ }^{6}$, Brian P. Lucas, MD, MS ${ }^{6,7}$, Mahmoud El-Barbary, MD, PhD, MSc, \\ Society of Hospital Medicine Point of Care Ultrasound Task Force**, and Nilam J. Soni, MD, MS 10,11
}

\begin{abstract}
'Division of Hospital Medicine, University of North Carolina, Chapel Hill, North Carolina; 'Division of General Internal Medicine, University of Minnesota, Minneapolis, Minnesota; ${ }^{3}$ Division of Critical Care Medicine Services, Cooper Medical School of Rowan University, Camden, New Jersey; ${ }^{4}$ Division of General Internal Medicine, Medical College of Wisconsin, Milwaukee, Wisconsin; ${ }^{5}$ Department of Hospital Medicine, Kaiser Permanente San Francisco Medical Center, San Francisco, California; 'White River Junction VA Medical Center, White River Junction, Vermont; ${ }^{7}$ Geisel School of Medicine at Dartmouth College, Hanover, New Hampshire; ${ }^{8}$ King Saud Bin Abdulaziz University for Health Sciences, Riyadh, Saudi Arabia; ${ }^{9}$ Department of Clinical Epidemiology and Biostatistics, McMaster University, Hamilton, ON, Canada; ${ }^{10}$ Division of General \& Hospital Medicine, The University of Texas School of Medicine at San Antonio, San Antonio, Texas; ${ }^{11}$ Section of Hospital Medicine, South Texas Veterans Health Care System, San Antonio, Texas.
\end{abstract}

Executive Summary: 1) We recommend that ultrasound should be used to guide thoracentesis to reduce the risk of complications, the most common being pneumothorax. 2) We recommend that ultrasound guidance should be used to increase the success rate of thoracentesis. 3) We recommend that ultrasound-guided thoracentesis should be performed or closely supervised by experienced operators. 4) We suggest that ultrasound guidance be used to reduce the risk of complications from thoracentesis in mechanically ventilated patients. 5) We recommend that ultrasound should be used to identify the chest wall, pleura, diaphragm, lung, and subdiaphragmatic organs throughout the respiratory cycle before selecting a needle insertion site. 6) We recommend that ultrasound should be used to detect the presence or absence of an effusion and approximate the volume of pleural fluid to guide clinical decision-making. 7) We recommend that ultrasound should be used to detect complex sonographic features, such as septations, to guide clinical decision-making regarding the timing and method of pleural drainage. 8) We suggest that ultrasound be used to measure the depth from the skin surface to the parietal pleura to help select an appropriate length needle and determine the maximum needle insertion depth. 9) We suggest that ultrasound be used to evaluate normal lung sliding pre- and postprocedure to rule out pneumothorax. 10) We suggest avoiding delay or interval change in patient position from the time of marking the needle insertion site to performing the thoracentesis. 11) We recommend against performing routine postprocedure chest radiographs in patients who have undergone thoracentesis successfully with ultrasound guidance and are asymptomatic with normal lung sliding postprocedure. 12) We recommend that novices who use ultrasound guidance for thoracentesis should receive focused training in lung and pleural ultrasonography and hands-on practice in procedural technique. 13) We suggest that novices undergo simulation-based training prior to performing ultrasound-guided thoracentesis on patients. 14) Learning curves for novices to become competent in lung ultrasound and ultrasound-guided thoracentesis are not completely understood, and we recommend that training should be tailored to the skill acquisition of the learner and the resources of the institution. Journal of Hospital Medicine 2018;13:126-135. @ 2018 Society of Hospital Medicine

\begin{abstract}
*Address for correspondence and reprint requests: Ria Dancel, MD, FACP, FHM, FAAP, Division of Hospital Medicine, University of North Carolina, 101 Manning Drive, Campus Box 7085, Chapel Hill, NC 27599-7085; Telephone: (984) 974-1931; Fax: (984) 974-2216; E-mail: Ria.Dancel@unchealth.unc.edu
\end{abstract} ${ }^{\star *} A$ complete list of the Society of Hospital Medicine Point-of-Care Ultra-
sound Task Force members appears at the end of this article.

Additional Supporting Information may be found in the online version of this article.

Received: May 10, 2017; Revised: November 22, 2017;

Accepted: December 2, 2017

2018 Society of Hospital Medicine DOI 10.12788/jhm.2940

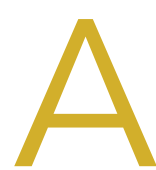

pproximately 1.5 million people develop a pleural effusion in the United States annually, and approximately 173,000 people (12\%) undergo thoracentesis. ${ }^{1}$ A recent review of thoracenteses performed at 234 University Health System Consortium hospitals between January 2010 and September 2013 demonstrated that 16\% of 132,472 thoracenteses were performed by general internists and hospitalists, $33.1 \%$ were performed by interventional radiologists, and $20.3 \%$ were performed by pulmonologists. ${ }^{2}$ The iatrogenic pneumothorax rate was not significantly different between interventional radiologists and internists $(2.8 \%$ and 
$2.9 \%$ risk, respectively); however, the admissions associated with bedside thoracentesis were less expensive than the admissions associated with thoracentesis performed in radiology suites, even after controlling for clinical covariates. ${ }^{2}$ In addition, the use of ultrasound guidance has been associated with a reduced risk of complications and cost of thoracentesis. ${ }^{3,4}$ In most of the early published studies on ultrasound-guided thoracentesis, the procedures were performed by radiologists. ${ }^{5-12}$ However, in 2010, the British Thoracic Society published guidelines on pleural procedures and thoracic ultrasound geared toward any trained provider. ${ }^{13}$ The purpose of this guideline is to review the literature and present evidence-based recommendations on the performance of ultrasound-guided thoracentesis at the bedside.

\section{METHODS}

Detailed methods are described in Appendix 1. The Society of Hospital Medicine (SHM) Point-of-care Ultrasound (POCUS) Task Force was assembled to carry out this guideline development project under the direction of the SHM Board of Directors, Director of Education, and Education Committee. All expert panel members were physicians or advanced practice providers with expertise in POCUS. The expert panel members were divided into working group members, external peer reviewers, and a methodologist. All the Task Force members were required to disclose any potential conflicts of interests (Appendix 2). The literature search was conducted in two independent phases. The first phase included literature searches conducted by the four working group members themselves. Key clinical questions were prepared prior to conducting a systematic literature search by a medical librarian. The Medline, Embase, CINAHL, and Cochrane medical databases were searched from 1975 to September 2015 initially. Updated searches were conducted in November 2016 and in August 2017 (Appendix 3). All article abstracts were first screened for relevance by at least two members of the working group. Full-text versions of the screened articles were reviewed, and the articles focusing on the use of ultrasound to guide thoracentesis were selected. Articles that discussed thoracentesis without ultrasound guidance were excluded. In addition, the following article types were excluded: non-English language, nonhuman, subjects' age $<18$ years, meeting abstracts, meeting posters, letters, and editorials. All relevant systematic reviews, meta-analyses, randomized controlled trials, and observational studies of ultrasound-guided thoracentesis were screened and selected. Final article selection was based on working group consensus, and the selected literature was incorporated into draft recommendations.

We used the RAND Appropriateness Method that required panel judgment and consensus. ${ }^{14}$ The 30 voting members of the SHM POCUS Task Force reviewed and voted on the draft recommendations considering the following five transforming factors: 1) Problem priority and importance, 2) Level of quality of evidence, 3) Benefit/harm balance, 4) Benefit/burden balance, and 5) Certainty/concerns about PEAF (Preferences/ Equity Acceptability/Feasibility). Panel members participated in two rounds of electronic voting using an internet-based electronic data collection tool (Redcap ${ }^{\mathrm{TM}}$ ) in December 2016 and January 2017 (Appendix 4). Voting on appropriateness was conducted using a 9-point Likert scale, and the degree of consensus was assessed using the RAND algorithm. Establishing a recommendation required at least $70 \%$ agreement and a strong recommendation required $80 \%$ agreement according to the RAND rules (Appendix 1, Figure 1). Disagreement was defined as $>30 \%$ of panelists voting outside of the zone of the median (appropriate, uncertain, inappropriate).

Recommendations were classified as strong or weak/conditional based on preset rules defining the panel's level of consensus, which determined the wording for each recommendation (Appendix 1, Table 2). The revised consensus-based recommendations underwent internal and external review by POCUS experts from different subspecialties. The final review of the guideline document was performed by all the members of the SHM POCUS Task Force, the SHM Education Committee, and the SHM Board of Directors. The SHM Board of Directors endorsed the document prior to submission to the Journal of Hospital Medicine.

\section{RESULTS}

\section{Literature search}

A total of 1,556 references were pooled from the following four different sources: a search by a certified librarian in September 2015 (1066 citations) that was updated in November 2016 (165 citations) and again in August 2017 (9 citations), working group members' literature searches (47 citations), and a search focused on training (269 citations). The final selection included 94 articles that were abstracted into a data table and incorporated into the draft recommendations. The details of the literature search strategy are given in Appendix 3.

\section{Recommendations}

Four domains (clinical outcomes, technique, training, and knowledge gaps) with $20 \mathrm{draft}$ recommendations were generated based on an initial review of the literature. The quality of evidence was appraised after assigning references to each draft recommendation. After two rounds of panel voting, five recommendations did not achieve agreement based on the RAND rules (failure of achieving a threshold of at least $70 \%$ and/or uncertainty expressed by panel median voting in the uncertain region), ${ }^{14}$ and 15 statements received final approval. The degree of consensus based on the median score and the dispersion of voting around the median are shown in Appendix 5. Ten statements were approved as strong recommendations, and five were approved as conditional recommendations. Recommendation 3 was deleted due to its similarity to the first two statements. This yielded a final recommendation count of 14 . For each recommendation, the strength of the recommendation and the degree of consensus are summarized in Table 1.

\section{Terminology}

- Thoracentesis is a procedure of aspiration of fluid from the pleural space by percutaneous insertion of a needle through 
the chest wall with or without the insertion of a catheter.

- In this document, ultrasound guidance refers to static guidance and site marking performed at the bedside immediately before the procedure, as opposed to real-time (dynamic) ultrasound guidance or radiology performed site marking. The static method is the most commonly used method of ultrasound guidance and is supported by current evidence.

\section{RECOMMENDATIONS}

\section{Clinical Outcomes}

\section{We recommend that ultrasound should be used to guide thoracentesis to reduce the risk of complica- tions, the most common being pneumothorax.}

Rationale: Both static ultrasound guidance and dynamic ultrasound guidance have been reported to be associated with a reduced risk of pneumothorax. ${ }^{4-7,15-18}$ A meta-analysis of 24 studies that included 6,605 thoracenteses showed a significant decrease in the risk of postprocedure pneumothorax with the use of ultrasound guidance compared to the risk associated with thoracentesis performed based on landmarks alone (OR $0.3,95 \% \mathrm{Cl}$ 0.2-0.7). ${ }^{3}$ The meta-analysis included both prospective and retrospective studies conducted using both static and dynamic ultrasound guidance. ${ }^{3}$ A large retrospective cohort study conducted by Mercaldi et al. comprising more than 61,000 patients who underwent thoracentesis also showed that ultrasound guidance was associated with reduced odds of pneumothorax (OR 0.8 [0.7-0.9]). ${ }^{4}$ When pneumothorax did occur during that hospitalization, the cost of hospitalization increased by $\$ 2800$ and the length of stay increased by 1.5 days. ${ }^{4}$ A 2008 review of 19,339 thoracenteses conducted by Patel et al. also demonstrated an association between ultrasound guidance and reduced odds of pneumothorax (OR 0.8 [0.7-0.96]). ${ }^{18}$ Although these findings were significant, it is important to note that the studies of both Mercaldi et al. and Patel et al. were reviews of administrative databases conducted using the International Classification of Diseases, 9th Revision (ICD-9) codes for thoracentesis and Current Procedure Terminology $-4^{\text {th }}$ edition (CPT) codes for the use of ultrasound. 4,18 Patel et al. identified pneumothorax using ICD-9 codes for "pneumothorax-iatrogenic" and "pneumothorax-not specified as due to the procedure." The association between ultrasound guidance and the reduced odds of pneumothorax was driven by the latter code. ${ }^{18}$ However, as with most retrospective studies using administrative data, granular data about the patients, procedure, proceduralists, and complications were not available in these reviews and conclusions may be limited by erroneous coding or documentation. ${ }^{4,18}$ In a third retrospective cohort study, Raptopoulos et al. compared 154 landmark-based thoracenteses performed by "clinical physicians" and 188 ultrasound-guided thoracenteses performed by radiologists and found that ultrasound-guided site selection reduced the rate of pneumothorax from $18 \%$ to $3 \%(P<.0001) .{ }^{6}$ Finally, one single-center randomized controlled trial of 160 thoracenteses performed by pulm- onologists showed that ultrasound guidance reduced the relative risk of pneumothorax by $90 \%(12.5 \%$ vs $1.3 \%$; $P=.009)$ with a number needed to treat of $9 .{ }^{15}$ It was not possible to blind the operators to the use of ultrasound guidance, but the data analysis was blinded. ${ }^{15}$ Furthermore, while there was no explicit comparison of the intervention vs. the control groups, randomization would have presumably rendered both groups similar in terms of patient characteristics and effusion characteristics. ${ }^{15}$ Ultrasound may reduce the risk of pneumothorax through several mechanisms, including identifying patients in whom thoracentesis cannot be safely performed, allowing selection of the safest needle insertion site, and revealing the optimal depth of needle insertion.

\section{We recommend that ultrasound guidance should be used to increase the success rate of thoracentesis.}

Rationale: Thoracentesis guided by ultrasound has lower rates of failed attempts, or "dry taps," compared to thoracentesis guided solely by physical examination. In 1977, Ravin described a method of using ultrasound to guide successful drainage of six complex pleural effusions (empyema or loculated effusion) after multiple (5-7) failed attempts by clinicians using physical examination alone. ${ }^{8}$ In a second study by radiologists, Weingardt et al. demonstrated that 20 of 26 failed landmark-based thoracenteses were due to incorrect site selection by physical examination-15 sites were below the diaphragm and 5 sites were above the pleural effusion or in the consolidated lung-and the use of ultrasound allowed successful sampling in 14 of 16 patients who had a failed landmark-based thoracentesis. ${ }^{9}$ Diacon et al. asked 30 physicians, ranging from junior housestaff to pulmonologists, to mark 172 potential thoracentesis sites in 67 patients with pleural effusions using physical examination alone. Ultrasound was then used to evaluate the proposed puncture sites. They found that using ultrasound would have avoided puncture on "dry chests" in $2 \%$ and avoided potential laceration of a solid organ in $10 \%$ of patients compared to site selection by physical examination alone. ${ }^{19}$ Finally, Perazzo et al. randomized 160 patients to landmark-based thoracentesis and ultrasound-guided thoracentesis and demonstrated that half of the eight dry taps that occurred in the control group could be successfully drained using subsequent ultrasound guidance. ${ }^{15}$

\section{Technique}

\section{We recommend that ultrasound-guided thoracentesis should be performed or closely supervised by experi- enced operators.}

Rationale: Current evidence suggests lower complication rates when thoracentesis is performed by experienced healthcare providers. A systematic review of 6,605 thoracenteses showed a significantly lower pneumothorax rate when thoracentesis was performed by pulmonology or radiology faculty versus resident physicians (3.9\% vs $8.5 \% ; P=.04$ ), although this finding was not significant in the four studies that directly com- 
TABLE 1. Summary of Recommendations

\begin{tabular}{|c|c|c|c|}
\hline No. & Topic of Recommendation & Strength of Recommendation & Degree of Consensus \\
\hline \multicolumn{4}{|c|}{ Clinical Outcomes } \\
\hline 1 & Risk of postprocedure pneumothorax & Strong & Very good \\
\hline \multirow[t]{2}{*}{2} & Thoracentesis procedure success rates & Strong & Very good \\
\hline & Risk of bleeding & N/A & N/A \\
\hline \multicolumn{4}{|c|}{ Technique } \\
\hline 3 & Operator experience needed & Strong & Very good \\
\hline 4 & Complications in mechanically ventilated patients & Conditional & Good \\
\hline 5 & Identification of critical structures & Strong & Very good \\
\hline 6 & Detect and approximate pleural fluid volume & Strong & Very good \\
\hline \multirow[t]{2}{*}{7} & Sonographic features guide management & Strong & Very good \\
\hline & Indications for additional imaging & N/A & N/A \\
\hline 8 & Measurement of fluid depth & Conditional & Good \\
\hline \multirow[t]{2}{*}{9} & Lung sliding preprocedure and postprocedure & Conditional & Good \\
\hline & Detection of vessels with Doppler ultrasound & N/A & N/A \\
\hline \multirow[t]{2}{*}{10} & Avoid position changes after marking & Conditional & Good \\
\hline & Real-time ultrasound guidance & N/A & N/A \\
\hline \multirow[t]{2}{*}{11} & Postprocedure chest $\mathrm{X}$-rays & Strong & Very good \\
\hline & Postprocedure ultrasound examination & N/A & N/A \\
\hline \multicolumn{4}{|c|}{ Training } \\
\hline 12 & Training in lung and pleural ultrasound & Strong & Very good \\
\hline 13 & Simulation practice before real patient & Conditional & Good \\
\hline 14 & Learner skill acquisition curves vary & Strong & Very good \\
\hline
\end{tabular}

Grayed out recommendations did not achieve consensus. Abbreviations: N/A, Statements without recommendations due to lack of agreement/uncertainty

pared this factor. ${ }^{3}$ In a quality improvement study performed by Duncan et al., pulmonology and critical care physicians combining multiple quality improvement initiatives to achieve and maintain competency decreased the rate of pneumothorax from $8.6 \%$ to $1.1 \%(P=.0034) .{ }^{20}$ Interventions included ultrasound training, performance of 10 thoracenteses under expert supervision, and restriction of privileges to proceduralists who perform 10 or more thoracenteses per year. ${ }^{20}$ Finally, a series of 9,320 ultrasound-guided thoracenteses performed or supervised by a single expert internist over a period of 12 years resulted in a pneumothorax rate of $0.6 \%$ and a composite complication rate of $0.98 \%$ (pneumothorax, reexpansion pulmonary edema, hemothorax, site bleeding, hematoma, splenic laceration, and vasovagal reaction). ${ }^{21}$ Notably, pneumothorax rate in resident physician hands was reported to be $8.5 \%$ in the meta-analysis performed by Gordon et al., which is similar to the initial rate in the pulmonologists who participated in the study by Duncan et al. 3,20 However, after instituting formal ultrasound training and other initiatives aimed at maintaining competency, the pneumothorax rate in the study by Duncan et al. decreased to $1.1 \%$, similar to the rate observed in the series by Ault et al. ${ }^{21}$ This suggests that training and supervision are necessary to achieve competency and reduce the rate of complications. ${ }^{3,20,21}$

\section{We suggest that ultrasound guidance be used to reduce the risk of complications from thoracentesis in mechanically ventilated patients.}

Rationale: The rest of this guideline refers to ultrasound-guided thoracentesis performed in spontaneously breathing patients; however, this recommendation is specific to mechanically ventilated patients. Two prospective observational studies have shown no increase in complications when ultrasound-guided thoracentesis is performed on mechanically ventilated patients compared to patients not 
receiving positive pressure ventilation. A feasibility study of 45 thoracenteses performed on ventilated patients reported no complications, ${ }^{22}$ whereas another study on 232 patients reported a pneumothorax rate of $1.3 \% .^{23}$ In a larger study conducted by Mayo et al., medicine housestaff performed thoracentesis under the supervision of intensivists who had undergone training in ultrasound prior to performing the procedure. ${ }^{23}$ In both studies, most of the patients were in a supine position, although positioning and puncture site were at the discretion of the physician, and both studies employed use of static ultrasound guidance. ${ }^{22,23}$ A large series of 9,320 ultrasound-guided thoracenteses that included 1,377 mechanically ventilated patients did not report a higher rate of pneumothorax $(0.8 \%)$ compared to that in spontaneously breathing patients $(0.61 \%) .{ }^{21}$ Finally, a meta-analysis of 19 observational studies comprising 1,124 mechanically ventilated patients who underwent pleural drainage procedures showed a low rate of pneumothorax (3.4\%) and hemothorax $(1.9 \%) .{ }^{24}$ Although the rate of complication was reported to be low in this meta-analysis, ultrasound was not employed in all studies and its use was not associated with a significant reduction in pneumothorax. ${ }^{24}$ This may be because 8 of the 19 studies used pigtail catheters or large-bore thoracostomy tubes which treat pneumothorax as they occur. ${ }^{24}$

\section{We recommend that ultrasound should be used to} identify the chest wall, pleura, diaphragm, lung, and subdiaphragmatic organs throughout the respiratory cycle before selecting a needle insertion site.

Rationale: The use of ultrasound improves the selection of a safe needle insertion site because sites chosen without ultrasound guidance may be below the diaphragm, over solid organs, 9,19 or in locations that risk puncture of the lung. ${ }^{9}$ Visualization of the chest wall, diaphragm, and lung, which define the boundaries of a pleural effusion, allows the clinician to confirm the presence of a drainable pleural effusion and assess for other pathologies, such as ascites and tumor, that may be mistaken for a pleural effusion. 22,25,26 Hypoechoic lesions can represent small loculated pleural effusions but also pleural plaques, pleural masses, peripheral lung masses, or abscesses. 27,28

\section{We recommend that ultrasound should be used to detect the presence or absence of an effusion and approximate the volume of pleural fluid to guide clinical decision-making.}

Rationale: The presence and approximate size of pleural fluid collections are important determinants of whether thoracentesis, another procedure, or no procedure should be performed. Ultrasonography has higher sensitivity and specificity for detecting pleural effusions and better differentiates effusions from consolidations compared with chest radiography. ${ }^{29-42}$ Ultrasound allows semiquantitative estimation of pleural fluid volume to determine whether thoracentesis should be performed. ${ }^{41-45}$ When using ultrasound to choose a site for thoracentesis, the British Thoracic Society Pleural Disease guidelines recommend $\geq 10 \mathrm{~mm}$ of pleural fluid between the visceral and parietal pleura. ${ }^{13}$ Pleural effusions of $<10-15 \mathrm{~mm}$ are considered too small to tap. ${ }^{22,23}$ In a prospective study of 45 patients, a measurement of $>9.9 \mathrm{~cm}$ by ultrasound between the chest wall and the "V-point," the intersection of the diaphragm and the collapsed lung, correlated with a pleural fluid volume of $>1$ liter. ${ }^{46}$ Another prospective study of 73 patients showed that a pleural effusion spanning $>3$ intercostal spaces by ultrasound also correlated with a pleural fluid volume of $>1$ liter. ${ }^{47}$ Anticipating the volume of fluid to be removed may aid in preplanning and procurement of larger capacity drainage containers prior to starting the procedure. Lung ultrasound can also change the management if the characteristic of the effusion suggests that an invasive procedure is unsafe or another diagnostic or therapeutic option is more appropriate. ${ }^{39}$ In a prospective cohort study of 189 mechanically ventilated patients, lung ultrasound guided the management in all patients with suspected effusion, leading to chest tube placement in 7 patients and thoracentesis in 34 patients. ${ }^{48}$

7. We recommend that ultrasound should be used to detect complex sonographic features, such as septations, to guide clinical decision-making regarding the timing and method of pleural drainage.

Rationale: Pleural effusions can be broadly categorized sonographically as simple or complex. Complex effusions are further categorized as with or without septation. Simple effusions are anechoic and are often, but not invariably, transudative..$^{49-51}$ The use of sonography and computerized tomography (CT) is complementary, but features of complex pleural effusions (fibrin stranding and septations) may be better visualized by ultrasound than by CT of the thorax. ${ }^{52}$ Detection of complex features should prompt the consideration of pleural fluid sampling. ${ }^{53,54}$ Exudative effusions from tuberculosis, malignancy, or other etiologies more often include debris, septations, or other complex features. ${ }^{55,56}$ Certain features such as a swirling debris, pleural thickening, and nodularity may be more often associated with malignancy, ${ }^{54,56}$ and advanced ultrasound techniques may be used to detect a trapped lung prior to attempting drainage of a malignant pleural effusion. ${ }^{57}$ Two studies found complex septated pleural effusions to be invariably exudative ${ }^{50,58}$ and drainage was unlikely to be successful without the placement of a chest tube.$^{50,58-60}$ Chest tube placement through fibrinolytic administration or video-assisted thoracoscopic surgery (VATS) may be more appropriate in the management of complex septated pleural effusions, ${ }^{59-61}$ and expert consultation with a thoracic specialist is recommended in these cases.

8. We suggest that ultrasound can be used to measure the depth from the skin surface to the parietal pleura to help select an appropriate length needle and determine the maximum needle insertion depth. 
Rationale: The distance from the skin to the parietal and visceral pleura can be measured by ultrasound to determine whether thoracentesis can be safely performed and to guide selection of an adequate length needle..$^{38}$ The length of needle required to penetrate the pleural space varies based on the thickness of the chest wall. Percussion of the chest wall is limited when there is more than $6 \mathrm{~cm}$ of subcutaneous tissue, ${ }^{62}$ making physical examination in obese patients unreliable for selecting an appropriate site or needle length for thoracentesis. Ultrasound allows visualization of deep soft tissues, well beyond the limits of percussion, and allows an accurate measurement of the chest wall. ${ }^{63}$

\section{We suggest that ultrasound can be used to evaluate normal lung sliding pre- and postprocedure to rule out pneumothorax.}

Rationale: Normal lung sliding indicates normal apposition and movement of visceral and parietal pleura and rules out pneumothorax with a sensitivity that exceeds that of chest radiography, according to a meta-analysis of 20 studies using computed tomography or escape of intrapleural air at the time of drainage as the gold standard. ${ }^{64}$ In this meta-analysis, the pooled sensitivity of ultrasound was reported to be $88 \%$ (85-91\%) compared to $52 \%$ (49-55\%) for radiography, although the analysis also suggests that the test characteristics are dependent on operator skill. ${ }^{64}$ However, although lung sliding rules out pneumothorax, absence of lung sliding is not specific for pneumothorax and other conditions, including pleural adhesions, pleurodesis, and bronchial obstruction, can cause the absence of lung sliding. ${ }^{64}$ Detection of a lung point conclusively rules in a pneumothorax. ${ }^{65}$ Provided that the preprocedure lung ultrasound examination revealed normal lung sliding, a postprocedure examination can be performed to effectively evaluate for pneumothorax. This modality does not use ionizing radiation, is less expensive than computed tomography, can be performed faster than bedside chest radiography, and is more sensitive than supine or upright chest radiography. ${ }^{64,66-71}$

\section{We suggest avoiding delay or interval change in pa- tient position between the time of marking the nee- dle insertion site and performing the thoracentesis.}

Rationale: Optimal patient positioning and ultrasound-guided site marking should be performed by the primary operator immediately before beginning an invasive procedure. Remote sonographic localization in which a radiologist marks a needle insertion site using ultrasound and the thoracentesis is performed at a later time by a different provider is an antiquated practice. Two early studies demonstrated that this practice is no safer than landmark-based thoracentesis. ${ }^{6,72}$ One prospective study of 205 patients performed in 1986 showed no significant decrease in the incidence of complications from thoracentesis performed using remote sonographic localization versus landmark-based drainage. ${ }^{72}$ Complications in that study included a total of 22 pneumothoraces and 1 hematoma. The rate of complications in the group of patients who had site marking performed by radiology faculty and subsequent thoracentesis by medicine housestaff or attending physicians was $9.7 \%$ versus a complication rate of $12.7 \%$ in the landmark-based group. ${ }^{72}$ In addition, Raptopoulos et al. observed no significant difference in the pneumothorax rate between 106 patients with landmark-based thoracenteses and 48 patients who were sonographically marked by radiology faculty and then returned to the ward for completion of the thoracentesis by medicine housestaff (19\% vs. 15\%, respectively). ${ }^{6}$ Both groups had significantly higher rates of pneumothorax compared to those who underwent thoracentesis performed using real-time ultrasound guidance by radiology trainees (3\%). ${ }^{6}$ The authors speculated that changing the patient's position shifted the position of the pleural effusion, ultimately leading to the reliance on physical examination for the tap site. ${ }^{6}$

\section{We recommend against performing routine} postprocedure chest radiographs in patients who have undergone thoracentesis successfully with ultrasound guidance and are asymptomatic with normal lung sliding postprocedure.

Rationale: Chest radiography post-thoracentesis is unlikely to add information that changes management, especially if performed routinely, but does add expense, radiation, and inconvenience. ${ }^{73}$ The most common serious complication of thoracentesis is pneumothorax, which is often accompanied by symptoms, particularly in those patients with pneumothorax large enough to warrant chest tube placement. ${ }^{10,74,75}$ Pihlajamaa et al. retrospectively studied 264 ultrasound-guided thoracenteses performed by radiologists or radiology residents and noted that of 11 pneumothoraces, only 1 necessitated chest tube placement. ${ }^{10}$ Aleman et al. prospectively studied 506 ultrasound-guided and physical examination-guided thoracenteses and found that only $1 \%$ of asymptomatic patients developed a pneumothorax. ${ }^{74}$ Eight of the 18 symptomatic patients required chest tube placement as opposed to 1 of the 488 asymptomatic patients. ${ }^{74}$ A large prospective study of 941 ultrasound-guided thoracentesis reported that only $0.3 \%$ of asymptomatic patients with no suspicion of pneumothorax required tube thoracostomy. ${ }^{5}$ Postprocedure chest radiographs may be considered when thoracentesis is performed on mechanically ventilated patients, particularly when high airway pressures exist. In a study of 434 patients undergoing thoracentesis, only 10 patients had a pneumothorax (2.3\%). ${ }^{11}$ Six of these pneumothoraces occurred in 92 mechanically ventilated patients (6.5\%), and 2 of these 6 patients required a chest tube. ${ }^{11}$ None of the 4 spontaneously breathing patients with pneumothorax required a chest tube. ${ }^{11}$

\section{Training}

12. We recommend that novices who use ultrasound guidance for thoracentesis should receive focused training in lung and pleural ultrasonography and hands-on practice in procedural technique. 
TABLE 2. Degree of Consensus, Strength of recommendation, and Wording

\begin{tabular}{lcc}
\hline Degree of consensus & Strength of recommendation & Wording [Function of voting] \\
\hline Perfect consensus & Strong & recommend - must/to be/will \\
\hline Very good consensus & Strong & recommend - should be/can \\
\hline Good consensus & Weak/Conditional & suggest - to do \\
\hline Some consensus & Weak/Conditional & suggest - may do \\
\hline No consensus Disagreement & NO & No recommendation was made regarding
\end{tabular}

Rationale: Healthcare providers have to gain various skills to safely perform ultrasound-guided thoracentesis independently. Trainees should learn how to use ultrasound to identify important structures (chest wall, ribs, lung, pleura, diaphragm, and subdiaphragmatic organs); detect pleural effusions with complex features, such as septations; identify consolidated lung tissue; and rule out a pneumothorax. Prospective studies done with novice learners have shown that focused training combining didactics and hands-on practice using simulation or live models improves skills to assess pleural effusions. ${ }^{76-84}$ Several additional procedural techniques such as patient positioning and needle insertion are also important but are beyond the scope of these guidelines.

\section{We suggest that novices undergo simulation-based training prior to performing ultrasound-guided thora- centesis on patients.}

Rationale: Simulation-based training for thoracentesis has been studied in providers with different levels of medical training, ranging from medical students and internal medicine residents to practicing pulmonologists. Studies suggest that training in a zero-risk environment with simulation task trainers leads to increased knowledge and skills without subjecting the patients to inexperienced operators. ${ }^{85-87}$ One study on simulator-based training in medical students showed skill retention at 6 months and these skills were at least partially transferred to increased competency on live patients. ${ }^{88}$ Checklists to train providers in ultrasound-guided thoracentesis have been published.89,90 An experiential training program for attending physicians that utilized task trainers, along with standardized equipment and procedural technique, resulted in a reduction in the pneumothorax rate from $8.6 \%$ to $1.1 \%{ }^{20}$

\section{Training curves for novices to become competent in} lung ultrasound and ultrasound-guided thoracentesis are not completely understood. We recommend that training should be tailored to the skill acquisition of the learner and the resources of the institution.

Rationale: Understanding the rates at which novices progress from performing procedures under direct supervision to performing them independently would be highly desirable to ensure patient safety, guide supervision, and maximize efficiency of training. However, there is limited research describing the rate of progression of learners through these stages, either with regard to time or number of procedures performed. Two studies have shown that with brief training programs, medical students ${ }^{88}$ and internal medicine residents ${ }^{87}$ can achieve high levels of proficiency to perform thoracentesis on simulators, which is durable over time; however, whether these findings in a simulated environment translate into clinically significant outcomes is largely unknown, and neither of these studies incorporated the use of ultrasound guidance in their training curricula. ${ }^{87,88}$ Another study of pulmonary and critical care physicians combined multiple quality improvement initiatives with a half day of ultrasound-guided thoracentesis training, a requirement to perform 10 supervised thoracenteses prior to independent practice, and an additional requirement to perform 10 thoracenteses per year to maintain privileges. ${ }^{20}$ These interventions resulted in a concentration of competency among a few proceduralists, decreasing the rate of pneumothorax from $8.6 \%$ to $1.1 \% .{ }^{20}$ Degradation of skills with disuse may also occur ${ }^{84}$; thus, procedures performed infrequently should at a minimum be subjected to increased supervision and/or retesting.

\section{KNOWLEDGE GAPS}

The process of developing these guidelines revealed important gaps in the literature regarding the use of ultrasound guidance for thoracentesis. First, it is uncertain whether the use of ultrasound reduces the risk of bleeding with thoracentesis. A retrospective cohort study of 19,339 thoracenteses suggests that ultrasound guidance is associated with a $38.7 \%$ relative reduction in the odds of hemorrhage, although this reduction did not reach statistical significance (OR 0.6 [0.4-1.04]). ${ }^{18}$ Ultrasound may reduce the risk of bleeding by reducing the number of attempts and needle passes and potentially avoiding tortuous intercostal vessels, which can be found especially in elderly patients and more cephalad rib spaces. ${ }^{91}$ In an observational study of 22 patients undergoing thoracentesis, the intercostal artery (ICA) was identified by a high-frequency ultrasound transducer in 74 of 88 intercostal spaces..$^{92}$ The ICA is more exposed in the intercostal space within the first $6 \mathrm{~cm}$ lateral to the spinous processes and can be seen as far lateral as 
the midaxillary line. ${ }^{92-95}$ Thus, the ICA will most likely be avoided if a procedure site is selected $>6 \mathrm{~cm}$ lateral to the spinous processes and the needle is inserted above the rib.

Second, although all three studies conducted using real-time (dynamic) ultrasound guidance reported a pneumothorax rate of $<1 \%$, it is uncertain whether real-time ultrasound guidance confers any additional benefit compared to static guidance for site marking as direct comparisons were not made. ${ }^{17,96,97}$ It is possible that real-time ultrasound guidance may be superior to static guidance in certain situations, such as small pleural effusions of $<10-15 \mathrm{~mm}$ that have historically been considered too small to tap. ${ }^{13,22,23,96}$

Third, although one study suggests that general internists can safely perform thoracentesis with low complication rates similar to those of interventional radiologists, ${ }^{2}$ limited data exists on how to train practicing hospitalists to use ultrasound to guide thoracentesis. The effectiveness of different training protocols to acquire competence in ultrasound-guided thoracentesis has not been compared.

Finally, the impact of ultrasound use on patient experience has yet to be explored.

\section{CONCLUSION}

The use of ultrasound guidance for thoracentesis has been associated with increased success rates and decreased complication rates. Ultrasound can be used to estimate the pleural fluid volume, characterize the effusion as simple or complex, identify an optimal needle insertion site, and reduce the need for postprocedural chest radiographs. Training and experience are essential to reap the benefits of using ultrasound for thoracentesis, although our understanding of optimal educational strategies and learning curves is limited. Once training has occurred and competence is achieved, hospitalists can perform ultrasound-guided thoracentesis as safely as radiologists, pulmonologists, and other specialists.

\section{References}

1. Owings MF, Kozak LJ. Ambulatory and inpatient procedures in the United States, 1996. Vital Health Stat 13. 1998;139:1-119.

2. Kozmic SE, Wayne DB, Feinglass J, Hohmann SF, Barsuk JH. Factors associated with inpatient thoracentesis procedure quality at university hospitals. Jt Comm J Qual Patient Saf. 2016;42(1):34-40.

3. Gordon CE, Feller-Kopman D, Balk EM, Smetana GW. Pneumothorax following thoracentesis: a systematic review and meta-analysis. Arch Intern Med. 2010;170(4):332-339.

4. Mercaldi CJ, Lanes SF. Ultrasound guidance decreases complications and improves the cost of care among patients undergoing thoracentesis and paracentesis. Chest. 2013;143(2):532-538.

5. Jones PW, Moyers JP, Rogers JT, Rodriguez RM, Lee YC, Light RW. Ultrasound-guided thoracentesis: is it a safer method? Chest. 2003;123(2): 418-423.

6. Raptopoulos V, Davis LM, Lee G, Umali C, Lew R, Irwin RS. Factors affecting the development of pneumothorax associated with thoracentesis. AJR Am J Roentgenol. 1991;156(5):917-920.

7. Grogan DR, Irwin RS, Channick R, et al. Complications associated with thoracentesis. A prospective, randomized study comparing three different methods. Arch Intern Med. 1990;150(4):873-877.

8. Ravin CE. Thoracocentesis of loculated pleural effusions using grey scale ultrasonic guidance. Chest. 1977;71(5):666-668.

9. Weingardt JP, Guico RR, Nemcek AA, Jr., Li YP, Chiu ST. Ultrasound findings following failed, clinically directed thoracenteses. J Clin Ultrasound. 1994;22(7):419-426

\section{Acknowledgment}

Collaborators from the Society of Hospital Medicine Point-of-care Ultrasound Task Force: Saaid Abdel-Ghani, Robert Arntfield, Jeffrey Bates, Anjali Bhagra, Michael Blaivas, Daniel Brotman, Carolina Candotti, Richard Hoppmann, Susan Hunt, Trevor P. Jensen, Venkat Kalidindi, Ketino Kobaidze, Joshua Lenchus, Benji Mathews, Paul, Mayo, Satyen Nichani, Vicki Noble, Martin Perez, Aliaksei Pustavoitau, Kreegan Reierson, Sophia Rodgers, Gerard Salame, Kirk Spencer, Vivek Tayal, David M. Tierney.

Disclosures: Ricardo Franco-Sadud reports institutional funds received from the Society of Hospital Medicine Annual Meeting for travel expenses and accommodations outside the submitted work. Nitin Puri reports Payment for lectures including service on speakers bureaus from Fujifilm Sonosite and royalties from Elsevier, both outside the submitted work. All other authors have nothing to disclose.

Funding: Brian P Lucas: Department of Veterans Affairs, Veterans Health Administration, Office of Research and Development and Dartmouth SYNERGY, National Institutes of Health, National Center for Translational Science (UL1TR001086). Nilam Soni: Department of Veterans Affairs, Quality Enhancement Research Initiative (QUERI) Partnered Evaluation Initiative Grant (HX002263-01A1)

Disclaimer: The contents of this publication do not represent the views of the U.S. Department of Veterans Affairs or the United States Government.

The authors thank all the members of the Society of Hospital Medicine Pointof-care Ultrasound Task Force and the Education Committee members for their time and dedication to develop these guidelines.

CHAIRS: Nilam Soni, Ricardo Franco Sadud, Jeff Bates. WORKING GROUPS: Thoracentesis Working Group: Ria Dancel (chair), Daniel Schnobrich, Nitin Puri. Vascular Access Working Group: Ricardo Franco (chair), Benji Matthews, Saaid Abdel-Ghani, Sophia Rodgers, Martin Perez, Daniel Schnobrich. Paracentesis Working Group: Joel Cho (chair), Benji Matthews, Kreegan Reierson, Anjali Bhagra, Trevor P. Jensen. Lumbar puncture Working Group: Nilam Soni (chair), Ricardo Franco, Gerard Salame, Josh Lenchus, Venkat Kalidindi, Ketino Kobaidze. Credentialing Working Group: Brian P Lucas (chair), David Tierney, Trevor P. Jensen. PEER REVIEWERS: Robert Arntfield, Michael Blaivas, Richard Hoppmann, Paul Mayo, Vicki Noble, Aliaksei Pustavoitau, Kirk Spencer, Vivek Tayal. METHODOLOGIST: Mahmoud El Barbary. LIBRARIAN: Loretta Grikis. SOCIETY OF HOSPITAL MEDICINE EDUCATION COMMITTEE: Dan Brotman (past chair), Satyen Nichani (current chair), Susan Hunt. SOCIETY OF HOSPITAL MEDICINE STAFF: Nick Marzano.

10. Pihlajamaa K, Bode MK, Puumalainen T, Lehtimaki A, Marjelund S, Tikkakoski T. Pneumothorax and the value of chest radiography after ultrasound-guided thoracocentesis. Acta Radiol. 2004;45(8):828-832.

11. Gervais DA, Petersein A, Lee MJ, Hahn PF, Saini S, Mueller PR. US-guided thoracentesis: requirement for postprocedure chest radiography in patients who receive mechanical ventilation versus patients who breathe spontaneously. Radiology. 1997;204(2):503-506.

12. Boland GW, Gazelle GS, Girard MJ, Mueller PR. Asymptomatic hydropneumothorax after therapeutic thoracentesis for malignant pleural effusions. AJR Am J Roentgenol. 1998;170(4):943-946.

13. Havelock T, Teoh R, Laws D, Gleeson F. Pleural procedures and thoracic ultrasound: British Thoracic Society Pleural Disease Guideline 2010. Thorax. 2010;65(2):ii61-76.

14. Fitch K, Bernstein SJ, Aguilar MD, Burnand B, LaCalle JR. The RAND/UCLA appropriateness method user's manual. DTIC Document; 2001.

15. Perazzo A, Gatto P, Barlascini C, Ferrari-Bravo M, Nicolini A. Can ultrasound guidance reduce the risk of pneumothorax following thoracentesis? J Bras Pneumol. 2014;40(1):6-12.

16. Barnes TW, Morgenthaler TI, Olson EJ, Hesley GK, Decker PA, Ryu JH. Sonographically guided thoracentesis and rate of pneumothorax. J Clin Ultrasound. 2005;33(9):442-446.

17. Cavanna L, Mordenti P, Berte R, et al. Ultrasound guidance reduces pneumothorax rate and improves safety of thoracentesis in malignant pleural effusion: report on 445 consecutive patients with advanced cancer. World J Surg Oncol. 2014;12:139.

18. Patel PA, Ernst FR, Gunnarsson CL. Ultrasonography guidance reduces com- 
plications and costs associated with thoracentesis procedures. J Clin Ultrasound. 2012:40(3):135-141.

19. Diacon $A H$, Brutsche $M H$, Soler M. Accuracy of pleural puncture sites: a prospective comparison of clinical examination with ultrasound. Chest. 2003;123(2):436-441.

20. Duncan DR, Morgenthaler TI, Ryu JH, Daniels CE. Reducing iatrogenic risk in thoracentesis: establishing best practice via experiential training in a zero-risk environment. Chest. 2009;135(5):1315-1320.

21. Ault MJ, Rosen BT, Scher J, Feinglass J, Barsuk JH. Thoracentesis outcomes: a 12-year experience. Thorax. 2015;70(2):127-132

22. Lichtenstein D, Hulot JS, Rabiller A, Tostivint I, Meziere G. Feasibility and safety of ultrasound-aided thoracentesis in mechanically ventilated patients. Intensive Care Med. 1999;25(9):955-958

23. Mayo PH, Goltz HR, Tafreshi M, Doelken P. Safety of ultrasound-guided thoracentesis in patients receiving mechanical ventilation. Chest. 2004;125(3):1059-1062

24. Goligher EC, Leis JA, Fowler RA, Pinto R, Adhikari NK, Ferguson ND. Utility and safety of draining pleural effusions in mechanically ventilated patients: a systematic review and meta-analysis. Crit Care. 2011;15(1):R46.

25. Landay M, Harless W. Ultrasonic differentiation of right pleural effusion from subphrenic fluid on longitudinal scans of the right upper quadrant: importance of recognizing the diaphragm. Radiology. 1977;123(1):155-158.

26. Mayo PH, Doelken P. Pleural ultrasonography. Clin Chest Med. 2006;27(2): 215-227.

27. Rosenberg ER. Ultrasound in the assessment of pleural densities. Chest. 1983;84(3):283-285

28. Gorg C, Restrepo I, Schwerk WB. Sonography of malignant pleural effusion. Eur Radiol. 1997;7(8):1195-1198

29. Gryminski J, Krakowka P, Lypacewicz G. The diagnosis of pleural effusion by ultrasonic and radiologic techniques. Chest. 1976;70(1):33-37

30. Kalokairinou-Motogna M, Maratou K, Paianid I, et al. Application of color Doppler ultrasound in the study of small pleural effusion. Med Ultrason. 2010;12(1):12-16.

31. Lichtenstein D, Goldstein I, Mourgeon E, Cluzel P, Grenier P, Rouby JJ. Comparative diagnostic performances of auscultation, chest radiography, and lung ultrasonography in acute respiratory distress syndrome. Anesthesiology. 2004;100(1):9-15.

32. Grimberg A, Shigueoka DC, Atallah AN, Ajzen S, lared W. Diagnostic accuracy of sonography for pleural effusion: systematic review. Sao Paulo Med J. 2010;128(2):90-95.

33. Kataoka H. Utility of thoracic sonography for follow-up examination of chronic heart failure patients with previous decompensation. Clin Cardiol. 2007;30(7):336-341.

34. Ma OJ, Mateer JR. Trauma ultrasound examination versus chest radiography in the detection of hemothorax. Ann Emerg Med. 1997;29(3):312-315.

35. Rocco M, Carbone I, Morelli A, et al. Diagnostic accuracy of bedside ultrasonography in the ICU: feasibility of detecting pulmonary effusion and lung contusion in patients on respiratory support after severe blunt thoracic trauma. Acta Anaesthesiol Scand. 2008;52(6):776-784

36. Kocijancic I, Vidmar K, Ivanovi-Herceg Z. Chest sonography versus latera decubitus radiography in the diagnosis of small pleural effusions. J Clin UI trasound. 2003;31(2):69-74.

37. Lichtenstein DA. Lung ultrasound in the critically ill. Ann Intensive Care. 2014;4(1):1.

38. Soni NJ, Franco R, Velez MI, et al. Ultrasound in the diagnosis and management of pleural effusions. J Hosp Med. 2015;10(12):811-816.

39. Medford AR, Entwisle JJ. Indications for thoracic ultrasound in chest medicine: an observational study. Postgrad Med J. 2010;86(1011):8-11.

40. Lin MS, Hwang JJ, Chong IW, et al. Ultrasonography of chest diseases: analysis of 154 cases. Gaoxiong Yi Xue Ke Xue Za Zhi . 1992;8(10):525-534.

41. Eibenberger KL, Dock WI, Ammann ME, Dorffner R, Hormann MF, Grabenwoger F. Quantification of pleural effusions: sonography versus radiography. Radiology. 1994;191(3):681-684

42. Vignon $P$, Chastagner $C$, Berkane $V$, et al. Quantitative assessment of pleural effusion in critically ill patients by means of ultrasonography. Crit Care Med. 2005;33(8):1757-1763

43. Usta E, Mustafi M, Ziemer G. Ultrasound estimation of volume of postoperative pleural effusion in cardiac surgery patients. Interact Cardiovasc Thorac Surg. 2010;10(2):204-207

44. Remerand F, Dellamonica J, Mao Z, et al. Multiplane ultrasound approach to quantify pleural effusion at the bedside. Intensive Care Med. 2010;36(4):656-664.

45. Balik M, Plasil P, Waldauf $P$, et al. Ultrasound estimation of volume of pleural fluid in mechanically ventilated patients. Intensive Care Med. 2006;32(2): 318-321.
46. Zanforlin A, Gavelli G, Oboldi D, Galletti S. Ultrasound-guided thoracentesis: the V-point as a site for optimal drainage positioning. Eur Rev Med Pharmacol Sci. 2013;17(1):25-28.

47. Lisi M, Cameli M, Mondillo S, et al. Incremental value of pocket-sized imaging device for bedside diagnosis of unilateral pleural effusions and ultrasound-guided thoracentesis. Interact Cardiovasc Thorac Surg. 2012;15(4):596-601.

48. Xirouchaki N, Kondili E, Prinianakis G, Malliotakis P, Georgopoulos D. Impact of lung ultrasound on clinical decision making in critically ill patients. Intensive Care Med. 2014;40(1):57-65.

49. Chen HJ, Tu CY, Ling SJ, et al. Sonographic appearances in transudative pleural effusions: not always an anechoic pattern. Ultrasound Med Biol. 2008;34(3):362-369.

50. Yang PC, Luh KT, Chang DB, Wu HD, Yu CJ, Kuo SH. Value of sonography in determining the nature of pleural effusion: analysis of 320 cases. AJR Am J Roentgenol. 1992;159(1):29-33.

51. Liang SJ, Tu CY, Chen HJ, et al. Application of ultrasound-guided pigtail catheter for drainage of pleural effusions in the ICU. Intensive Care Med. 2009;35(2):350-354.

52. McLoud TC, Flower CD. Imaging the pleura: sonography, CT, and MR imaging. AJR Am J Roentgenol. 1991;156(6):1145-1153.

53. Tu CY, Hsu WH, Hsia TC, et al. Pleural effusions in febrile medical ICU patients: chest ultrasound study. Chest. 2004;126(4):1274-1280.

54. Sajadieh H, Afzali F, Sajadieh V, Sajadieh A. Ultrasound as an alternative to aspiration for determining the nature of pleural effusion, especially in older people. Ann N Y Acad Sci. 2004;1019:585-592.

55. Marcun R, Sustic A. Sonographic evaluation of unexplained pleural exudate: a prospective case series. Wien Klin Wochenschr. 2009;121(9-10):334-338.

56. Bugalho A, Ferreira D, Dias SS, et al. The diagnostic value of transthoracic ultrasonographic features in predicting malignancy in undiagnosed pleural effusions: a prospective observational study. Respiration. 2014;87(4):270-278.

57. Salamonsen MR, Lo AK, Ng AC, Bashirzadeh F, Wang WY, Fielding DI. Novel use of pleural ultrasound can identify malignant entrapped lung prior to effusion drainage. Chest. 2014;146(5):1286-1293

58. Himelman RB, Callen PW. The prognostic value of loculations in parapneumonic pleural effusions. Chest. 1986;90(6):852-856

59. Chen $\mathrm{CH}$, Chen W, Chen HJ, et al. Transthoracic ultrasonography in predicting the outcome of small-bore catheter drainage in empyemas or complicated parapneumonic effusions. Ultrasound Med Biol. 2009;35(9):1468-1474.

60. Hirsch JH, Rogers JV, Mack LA. Real-time sonography of pleural opacities. AJR Am J Roentgenol. 1981;136(2):297-301.

61. Chen KY, Liaw YS, Wang HC, Luh KT, Yang PC. Sonographic septation: a useful prognostic indicator of acute thoracic empyema. J Ultrasound Med. 2000;19(12):837-843

62. Diaz-Guzman E, Budev MM. Accuracy of the physical examination in evaluating pleural effusion. Cleve Clin J Med. 2008;75(4):297-303.

63. Rhyne T, Birnholz JC. Simple measurement of chest-wall thickness with ultrasound. Radiology. 1973;108(2):436-438

64. Ding W, Shen Y, Yang J, He X, Zhang M. Diagnosis of pneumothorax by radiography and ultrasonography: a meta-analysis. Chest. 2011;140(4):859-866.

65. Lichtenstein D, Meziere G, Biderman P, Gepner A. The "lung point": an ultrasound sign specific to pneumothorax. Intensive Care Med. 2000;26(10): 1434-1440

66. Shostak E, Brylka D, Krepp J, Pua B, Sanders A. Bedside sonography for detection of postprocedure pneumothorax. J Ultrasound Med. 2013;32(6): 1003-1009

67. Alrajab S, Youssef AM, Akkus NI, Caldito G. Pleural ultrasonography versus chest radiography for the diagnosis of pneumothorax: review of the literature and meta-analysis. Crit Care. 2013;17(5):R208.

68. Alrajhi K, Woo MY, Vaillancourt C. Test characteristics of ultrasonography for the detection of pneumothorax: a systematic review and meta-analysis. Chest. 2012;141(3):703-708.

69. Sartori S, Tombesi P, Trevisani L, Nielsen I, Tassinari D, Abbasciano V. Accuracy of transthoracic sonography in detection of pneumothorax after sonographically guided lung biopsy: prospective comparison with chest radiography. AJR Am J Roentgenol. 2007;188(1):37-41.

70. Blaivas M, Lyon M, Duggal S. A prospective comparison of supine chest radiography and bedside ultrasound for the diagnosis of traumatic pneumothorax. Acad Emerg Med. 2005;12(9):844-849.

71. Lichtenstein DA, Meziere G, Lascols N, et al. Ultrasound diagnosis of occult pneumothorax. Crit Care Med. 2005;33(6):1231-1238.

72. Kohan JM, Poe $\mathrm{RH}$, Israel $\mathrm{RH}$, et al. Value of chest ultrasonography versus decubitus roentgenography for thoracentesis. Am Rev Respir Dis. 1986;133(6):1124-1126 
73. Capizzi SA, Prakash UB. Chest roentgenography after outpatient thoracentesis. Mayo Clin Proc. 1998;73(10):948-950.

74. Aleman C, Alegre J, Armadans L, et al. The value of chest roentgenography in the diagnosis of pneumothorax after thoracentesis. Am J Med. 1999;107(4):340-343.

75. Petersen WG, Zimmerman R. Limited utility of chest radiograph after thoracentesis. Chest. 2000;117(4):1038-1042.

76. Begot E, Grumann A, Duvoid T, et al. Ultrasonographic identification and semiquantitative assessment of unloculated pleural effusions in critically ill patients by residents after a focused training. Intensive Care Med. 2014;40(10):1475-1480.

77. Kotagal M, Quiroga E, Ruffatto BJ, et al. Impact of point-of-care ultrasound training on surgical residents' confidence. J Surg Educ. 2015;72(4):e82-87

78. Beaulieu $Y$, Laprise $R$, Drolet $P$, et al. Bedside ultrasound training using webbased e-learning and simulation early in the curriculum of residents. Crit UItrasound J. 2015;7:1

79. Schnobrich DJ, Olson AP, Broccard A, Duran-Nelson A. Feasibility and acceptability of a structured curriculum in teaching procedural and basic di agnostic ultrasound skills to internal medicine residents. J Grad Med Educ. 2013;5(3):493-497.

80. Chalumeau-Lemoine L, Baudel JL, Das V, et al. Results of short-term training of naive physicians in focused general ultrasonography in an intensive-care unit. Intensive Care Med. 2009;35(10):1767-1771.

81. Keddis MT, Cullen MW, Reed DA, et al. Effectiveness of an ultrasound training module for internal medicine residents. BMC Med Educ. 2011;11:75

82. Ramsingh D, Alexander B, Le K, Williams W, Canales C, Cannesson M. Comparison of the didactic lecture with the simulation/model approach for the teaching of a novel perioperative ultrasound curriculum to anesthesiology residents. J Clin Anesth. 2014;26(6):443-454

83. Sekiguchi H, Bhagra A, Gajic O, Kashani KB. A general Critical Care Ultrasonography workshop: results of a novel Web-based learning program combined with simulation-based hands-on training. J Crit Care. 2013;28(2):217.e217-212.

84. Dulohery MM, Stoven S, Kurklinsky AK, Halvorsen A, McDonald FS, Bhagra A. Ultrasound for internal medicine physicians: the future of the physical examination. J Ultrasound Med. 2014;33(6):1005-1011.
85. Lenchus J, Issenberg SB, Murphy D, et al. A blended approach to invasive bedside procedural instruction. Med Teach. 2011;33(2):116-123.

86. Lenchus JD. End of the "see one, do one, teach one" era: the next generation of invasive bedside procedural instruction. J Am Osteopath Assoc. 2010;110(6):340-346

87. Wayne DB, Barsuk JH, O'Leary KJ, Fudala MJ, McGaghie WC. Mastery learning of thoracentesis skills by internal medicine residents using simulation technology and deliberate practice. J Hosp Med. 2008;3(1):48-54.

88. Jiang $\mathrm{G}$, Chen $\mathrm{H}$, Wang $\mathrm{S}$, et al. Learning curves and long-term outcome of simulation-based thoracentesis training for medical students. BMC Med Educ. 2011;11:39.

89. Salamonsen M, McGrath D, Steiler G, Ware R, Colt H, Fielding D. A new instrument to assess physician skill at thoracic ultrasound, including pleural effusion markup. Chest. 2013;144(3):930-934

90. Berg D, Berg K, Riesenberg LA, et al. The development of a validated checklist for thoracentesis: preliminary results. Am J Med Qual. 2013;28(3): 220-226.

91. Shurtleff E, Olinger A. Posterior intercostal artery tortuosity and collateral branch points: a cadaveric study. Folia Morphol. 2012;71(4):245-251.

92. Salamonsen M, Ellis S, Paul E, Steinke K, Fielding D. Thoracic ultrasound demonstrates variable location of the intercostal artery. Respiration. 2012;83(4):323-329.

93. Salamonsen M, Dobeli K, McGrath D, et al. Physician-performed ultrasound can accurately screen for a vulnerable intercostal artery prior to chest drainage procedures. Respirology. 2013;18(6):942-947

94. Helm EJ, Rahman NM, Talakoub O, Fox DL, Gleeson FV. Course and variation of the intercostal artery by CT scan. Chest. 2013;143(3):634-639.

95. Yoneyama H, Arahata M, Temaru R, Ishizaka S, Minami S. Evaluation of the risk of intercostal artery laceration during thoracentesis in elderly patients by using 3D-CT angiography. Intern Med. 2010;49(4):289-292.

96. Soldati G, Smargiassi A, Inchingolo R, Sher S, Valente S, Corbo GM. Ultrasound-guided pleural puncture in supine or recumbent lateral position - feasibility study. Multidiscip Respir Med. 2013;8(1):18.

97. Harnsberger HR, Lee TG, Mukuno DH. Rapid, inexpensive real-time directed thoracentesis. Radiology. 1983;146(2):545-546. 